\title{
Improving the Performance of the Link Parser
}

\author{
Y. Viswanatha Naidu, Anil Kumar Singh, Dipti Misra Sharma, Akshar Bharati \\ Language Technologies Research Centre \\ IIIT, Hyderabad, India \\ Email: \{vnaidu, anil\}@ research.iiit.ac.in \\ $\{$ dipti\}@iiit.ac.in
}

\begin{abstract}
The paper describes an approach to extend the coverage of a Link Grammar based parser on the constructions that are not being handled currently by the grammar. There are about thirty types of constructions which we have identified till now. In order to make Link Grammar handle these constructions, we introduce a preprocessor and a postprocessor. The idea is to handle such constructions via some analysis and transformations in a preprocessing phase before the sentence is given to the Link Parser and then by adding the missing links in the postprocessing phase. The main part of the paper discusses the constructions not handled by the parser and introduces rule based preprocessor and postprocessor. This simple and flexible approach is able to increase the coverage of the parser significantly and allows even a relatively naive user to improve the performance of the parser without disturbing the core grammar.
\end{abstract}

\section{INTRODUCTION}

Parsers play a major role in Machine Translation (MT) and, more generally, in a number of other Natural Language Processing (NLP) tasks like question answering systems, anaphora resolution etc. Accordingly, they should be sufficiently sophisticated to be able to handle any text input, irrespective of language style and register. However, due to the inherent complexity of the task, most parsing systems often fail to parse more casual texts accurately. In fact, they often encounter difficulty in parsing even formal text. We therefore need more reliable syntactic parsing of text irrespective of the register or style.

Link Grammar is a word based grammar ([1], [2]). It analyses sentences in terms of relationships between pairs of words. Historically, Link Parser is optimized for formal language and it often does not parse more casual text and sometimes even formal text. For instance, the parser is not able to parse phenomena like topicalization, extraposition, certain discourse elements (or sentence connectives), certain co-ordinate constructions, some indirect questions and other miscellaneous patterns, which were obtained after experiments on a large subset of a corpus.

This scope for improving the parser performance is the motivation for this paper. Although there are some other dependency parsers which are relatively more robust ([3], [4], [5], [6]), Link Parser is still in use, most notably for grammar checking in the word processor called AbiWord. Also, because of its rule based nature, it allows linguistically aware but computationally inexperienced users to increase the coverage of the parser. In our approach we do this without disturbing the core grammar. This decision is based on the realization that if the user is not very familiar with the intricacies of grammar (sometimes even if he is), it is possible that fixing one problem may create another problem. Hence, instead of disturbing the grammar, we introduce a preprocessor and a postprocessor, which are used to allow the parser to handle those sentences which are not handled by the original parser.

We first discuss how various constructions are not handled by the parser. Then we show how these constructions can be parsed after the addition of the preprocessor and the postprocessor. Section 2 is an introduction to the Link Parser. Section 3 presents a brief survey of the literature. Section 4 presents constructions that are not handled by the parser. In section 5, we introduce the preprocessor and the postprocessor and their role in the parser. In section 6, we present a summary of the results.

\section{LINK PARSER}

The Link Parser [1] is a syntactic parser, based on Link Grammar, in which every word is associated with a set of links, which have some associated suffixes to furnish various linguistic information like number etc. describing some properties of the word. In this kind of parsing, instead of constructing constituents in a tree like hierarchy, the parser basically performs analysis in terms of the relationship between pairs of words. Given a sentence, the parser assigns to it a syntactic structure, which consists of a set of labeled links connecting pairs of words. The link grammar is very closely related to dependency grammars that were formally expressed by Gaifman in 1965 ([7], [3], [1]). It uses knowledge about capitalization, numerical expressions, and a variety of punctuation symbols ${ }^{1}$. Figure 1 shows Link Grammar's output for a sample sentence.

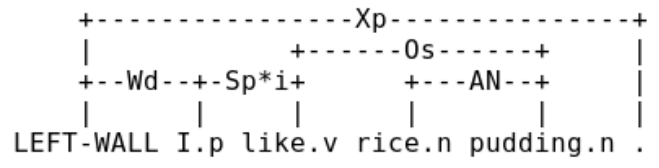

Fig. 1. Output of the Link Parser

In Figure 1 we see that $\mathbf{I}$ is linked on the right to the verb like with a link labeled $S p * i,{ }^{2}$ denoting Subject. (One can

\footnotetext{
${ }^{1}$ For more details see http://www.link.cs.cmu.edu/link/dict/

${ }^{2}$ Small letters represent gender, number, person etc.
} 
also say that like is linked on the left to I). Similarly, rice is linked on the right to the noun pudding with link labeled $A N$ denoting noun modifier to noun. And rice is linked on the left to the verb like with the link labeled $O s$, denoting Object. And $\mathbf{I}$ is linked on the left to the $\mathbf{\text { LEFT-WALL }}{ }^{3}$ with the link labelled $W d$.

The Link Parser also gives the phrase or the constituentstructure representation of a sentence. These structures are derived on the basis of the Link Grammar links. For the sentence shown in Figure 1, the constituent structure is shown in Figure 2.

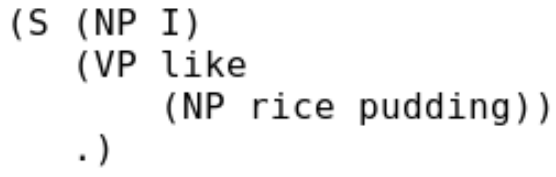

Fig. 2. Link Parser's constituent analysis

\section{BACKGROUND}

In this section we briefly present some background information on the Link Parser.

\section{A. A Small Note on Link Grammar}

The analysis given by the Link Parser is not always easy to interpret. Some of the labels are unlikely to be expected intuitively. To properly interpret we need to understand the entire system. This requires more effort from the user. Superficially, the analysis sometimes looks wrong even when it is not. For example, consider the parser's analysis of the sentence shown in Figure 3.

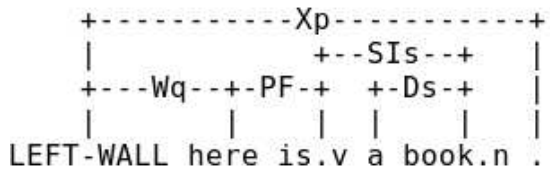

Fig. 3. An example of Link Parser's seemingly wrong analysis - 1

The parser gives a $W q$ label between here and $L E F T$-WALL. Note that $q$ in $W q$ may be misleading as $q$ is referring intuitively to question type label. Question type words, in fact, can get such a label, e.g. in Figure 4 Whom got a $W q$ label. In such a situation, one might wonder how can here be a question type word. The situation becomes more complex as even a preposition can have this label. See Figure 5 where Among gets a $W q$ link. To summarize, the same label can be assigned to different categories. To interpret the link labels, one has to understand the entire system. There is subject verb inversion (SIS) relation between is and book (in Figure 3) as given by the parser, although it is a declarative sentence. The

\footnotetext{
${ }^{3}$ The LEFT WALL is automatically inserted at the beginning of every sentence
}

meaning, according to parser's analysis, is that "SI" is used when the positions of the subject and verb are inverted, i.e., the verb comes first. That's the case here ("is" comes before "a book"). This inversion is different from the other subjectverb inversion like Will you go to the school?. In the latter case, will and you have been inverted.

This is just a small illustration of the complexity of the system from a user's point of view.

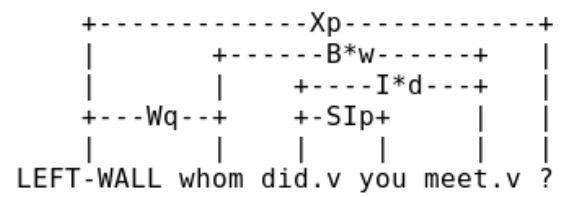

Fig. 4. Parser's analysis of a question sentence

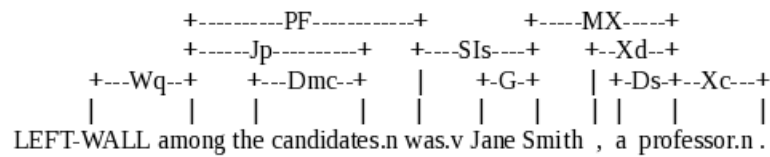

Fig. 5. Parser's seemingly wrong analysis - 2

\section{B. Making Changes in the Grammar}

Having illustrated the complexity of the Link Grammar we now propose that making changes inside the grammar is not an easy task for a common user. The Link Parser is now being maintained along with AbiWord, an open source word processor, which uses Link Grammar for grammar checking. Many changes and modifications are going on for improving the Link Parser. Understandably, since they are familiar with the grammar, their approach is to go inside the grammar and relaxing some conditions or making some other changes to improve the performance ${ }^{4}$. But this approach does not seem to work well because if we try to fix one problem by changing the grammar, it is very likely to create another problem somewhere else. This is the reason we chose not to disturb the existing grammar but to have an additional layer on top of it. We have some empirical evidence in support of this method. When we experimented on a corpus consisting of 2416 sentences on the recent version of the Link Parser and compared it with the old Link Parser ${ }^{5}$. The old Link Parser seems to show more consistent and better results than the new Link Parser. Details can be seen in Table I.

\footnotetext{
${ }^{4}$ For more details http://www.abisource.com/projects/link-grammar/ $\backslash \#$ download

${ }^{5}$ When we say the Link Parser or the old Link Parser we mean the CMU $4.41 \mathrm{~b}$ version. For more details http://www.link.cs.cmu.edu/link/ftp-site/ link-grammar/link-4.1b/. And when we say the new Link Parser, we mean the version link-grammar-4.4.3, developed by the AbiWord group from the old version. For more details see http://www.abisource.com/downloads/ link-grammar/.
} 


\begin{tabular}{|r|r|r|r|}
\hline & Handled & Unhandled & Coverage \\
\hline Old version & 1406 & 1010 & $58 \%$ \\
\hline New version & 1026 & 1390 & $42 \%$ \\
\hline
\end{tabular}

TABLE I

COMPARISON OF THE TWO VERSIONS

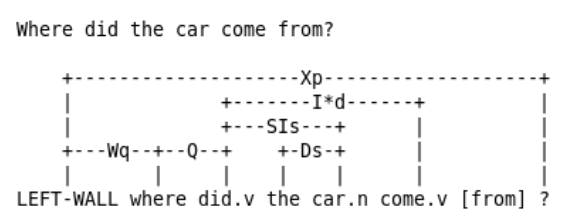

Fig. 6. Parser's analysis for preposition stranding

If it is not done, then do it.

$$
\text { [if] it is.v not done.v, then.e do.v it | }
$$

Fig. 7. Link Parser's analysis for the if-then clause

\section{B. If-Then Clauses}

The parser fails to parse the if-then clauses when the then clause subject is dropped. If we see Figure 7, it is clear that the parser was not able not handle it as it ignored the word if.

\section{What-If Conditionals}

The parser is also unable to handle the what if conditional clauses. If we look at Figure 8, it is obvious that the sentence was not fully parsed as the parser ignored both what and if.

\section{Discourse Connectives or Sentence Openers}

In the conversational text or even in some of the formal text, we often find many discourse connectives ([10], [11]) at the beginning of a sentence. Sample frequencies of some such connectives can be seen in Table II. When these sentences are passed through the Link Parser, it very often fails to parse them. For example sentences like Well it's the end of time. If

\begin{tabular}{|l|r|}
\hline Pattern & Frequency \\
\hline Well & 251387 \\
No & 85376 \\
Yes & 77573 \\
Here's & 26957 \\
Figure 1,2 ..8 & 19397 \\
What if & 10150 \\
think so & 9590 \\
such that & 2370 \\
Ladies gentlemen & 2248 \\
idea how & 1685 \\
face to face & 1530 \\
of even & 1345 \\
of conscience & 854 \\
peace of mind & 716 \\
guess so & 700 \\
In conclusion & 587 \\
frame of mind & 427 \\
as you like & 376 \\
speak so & 298 \\
But why not & 232 \\
walk so & 113 \\
\hline
\end{tabular}

TABLE II

FREQUENCY DISTRIBUTION OF UNPARSED PATTERNS

\footnotetext{
glish.

${ }^{9}$ Note: There were some other patterns which were sometimes handled by the parser and which we have not included in the list.

${ }^{6}$ Only when the sentence ends with from and if its object is where.

${ }^{7}$ For more details see http://www.link.cs.cmu.edu/link/dict/

${ }^{8}$ These frequencies are from the Corpus of Contemporary American En-
}

- Special constructions

- Conversational speech

- Lexicon might not be covered in the dictionary

We give some of the pattern frequencies ${ }^{8}$ from the corpus in Table II. As we can observe from this table ${ }^{9}$, it is clear that they are frequent enough. That the parser has failed to parse all of them shows the need for improvement.

\section{A. Preposition Stranding}

The parser is not able to parse a sentence when it ends with from and when it's object is where. From Figure 6 we can clearly see that not the whole of the sentence has been parsed. It is not the case that the parser does not handle all preposition stranding constructions in which a preposition with an object occurs somewhere other than immediately next to its object. For instance it can handle similar constructions with other stranded prepositions like Whom did you give the book $o$ ? 


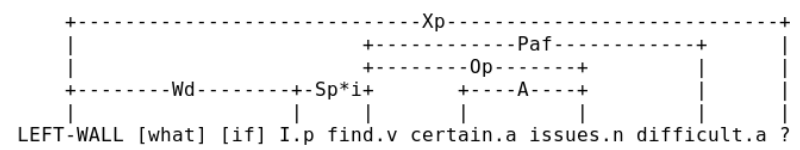

Fig. 8. Link Parser's analysis for the what if clause

Well it's the end of time.

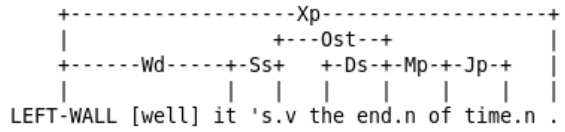

Fig. 9. Link Parser's analysis of discourse connectives - 1

we take a look at Figure 9, we can see that the parser has ignored the word well and performed the analysis for rest of the sentence. There is a similar problem with the sentence Yes, my personal request has not been met (See Figure 10). It ignores the word Yes and assigns structure to the rest of the sentence.

\section{E. Extraposition}

The sentence Details have emerged of a secret plan to finance the rebels. has not been parsed by the parser (See Figure 11). We can see that the parser did not parse emerged and of but interestingly handles the sentence when the noun is in its base position like Details of a secret plan to finance the rebels have emerged.

\section{F. Such-That Pattern}

The parser does not handle the sentences that have words such that (See Figure 12).

\section{G. Topicalization}

Topicalization is a phenomenon in which the focused expression appears in the sentence initial position. For example if we take the Figure 1 sentence, Rice pudding can be topicalized in the following manner: Rice pudding I like. The parser is not able to parse such a sentence.

\section{H. Miscellaneous Patterns}

The parser failed to parse the sentence If an experiment disagrees with the current theory, the theory has to be changed, not the experiment. We see in Figure 13 that the sentence was not fully parsed.

Similarly the sentence If the House agrees, I shall do as $\mathrm{Mr}$ Evans has suggested. was not parsed (See Figure 14).

Even when the preposition of is preceded by conscience the parser failed to parse (See Figure 15).

Still another construction is of the kind That John came to the party surprised Mary (See Figure 16).

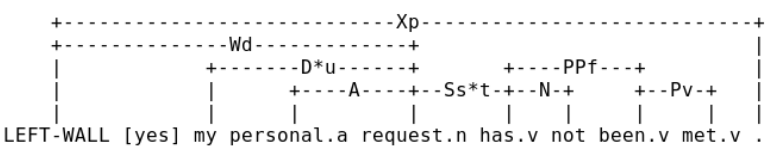

Fig. 10. Link Parser's analysis of discourse connectives - 2

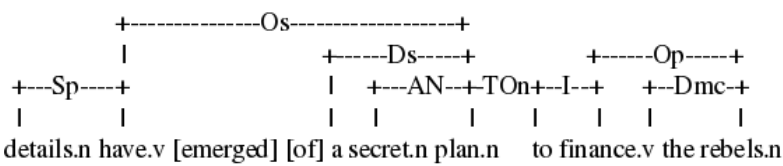

Fig. 11. Link parser' analysis for extraposition

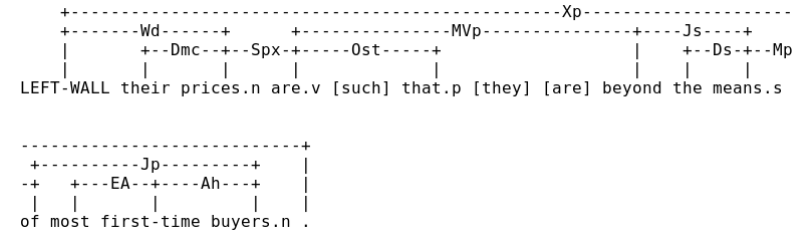

Fig. 12. Link Parser's analysis of such that construction

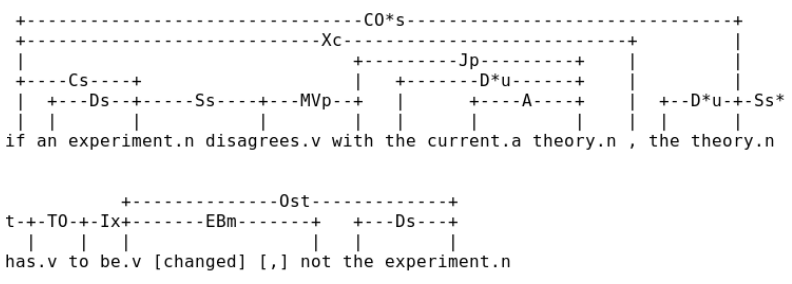

Fig. 13. Link parser's analysis of miscellaneous patterns - 1

If the House agrees, I shall do as Mr Evans has suggested.

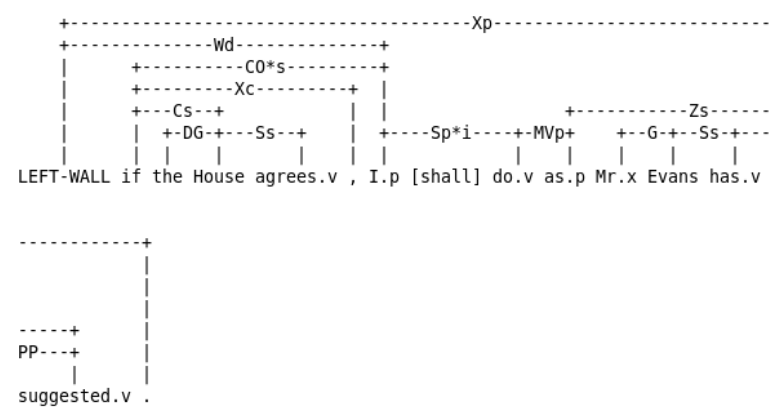

Fig. 14. Link Parser's analysis of miscellaneous patterns - 2

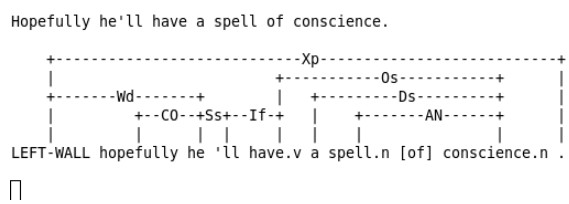

Fig. 15. Link parser's analysis of miscellaneous patterns - 3 


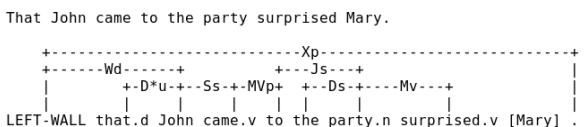

Fig. 16. Link parser's analysis of miscellaneous patterns - 4

\section{OUR APPROACH}

While discussing unhandled constructions we have also shown in section 2 that relaxing or changing the grammar may not be easy for the problematic sentences to be parsed. Instead it will actually decrease its coverage because unexpected effects occur elsewhere. Hence, we propose an approach which will not disturb the existing grammar but will still be an addition to grammar.

\section{A. Preprocessor}

The reason for introducing the preprocessor is to handle the cases that are not parsed by the parser. For instance if we take the construction types from 4.2 to 4.4 all of them are absolutely grammatical but the link grammar is not able to handle them. Hence the parser reports 'No complete linkages found'. However, in all the cases the parser failed to connect the sentence opener with the other words. They have the similar structures, i.e., the ignored words are occurring at the sentence initial position. One way is to consider all those sentences as two 'sentences', for which we have a rule like If the string begins with a 'Well', 'Yes', 'No', 'In conclusion' etc., split them into into two 'sentences' ${ }^{10}$. The first 'sentence' will be kept aside and the other will be given as an input to the parser. Accordingly, if-then clause type (section 4.2), what if types (section 4.3) and discourse connectives (section 4.4) will get split into two sentences. In the case of if then clauses if will be one sentence and the rest (e.g., it is not done, then do it.) as another sentence. In the case of what if, what if will be one sentence and the rest (I find certain issues difficult.) will be the other. In the case of discourse elements, in a sentence like Well, it's the end of time., the split will be as Well as one part and it's the end of time as the other part. After splitting the sentences, we will have the parse for Figures 8 as given in Figure 17. Once we get the parse of the sentence, we then add the first sentence to the parsed sentence in the postprocessing phase. Thus, the resulting parse will be as in Figure 18.

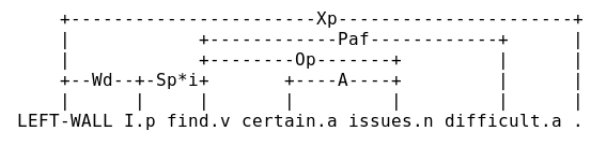

Fig. 17. Splitting sentences with what if conditionals

\footnotetext{
${ }^{10}$ Likewise, we list out all the possible discourse connectives ([10], [11]) that are present in the English language. If none of these are available, the preprocessor will not split the sentence.
}

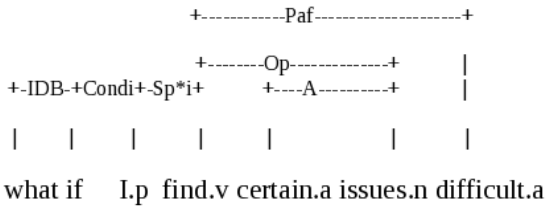

Fig. 18. Full parsed tree of the split sentence

Similarly, other discourse connectives like in conclusion, yes and so on will be handled with the split operation through preprocessing and postprocessing stages.

\section{B. Postprocessor}

The postprocessor that we use is similar to Link Grammar' dictionary $^{11}$. It can be seen as a tiny dictionary where we provide labels to the sentences that are not handled by the parser, based on the rules that exist in the preprocessor. It will have the words that do not exist in the original dictionary and for which grammar would be provided to get the analysis. This tiny dictionary does not conflict with the original dictionary grammar. Writing grammar here is easier as we do not need to understand the entire system of Link Grammar. Writing an extension to the grammar outside the main dictionary ${ }^{12}$ will not cause conflicts with the existing grammar (see Table III). In the postprocessor, the sentences that were splitted in the preprocessor phase will be joined in by the postprocessor along with link labels. For instance what if will be included in the dictionary that will be given a Condi+ link to the right indicated with + , and the sentences that are having discourse connective will be given an X- link to the left after the splitting operation, where $\mathrm{X}$ means a variable. In the case of Condi+, the X- will become Condi- link.

\begin{tabular}{|l|l|}
\hline Words & formula \\
\hline What_if & Condi+ \\
Well Yes No & $\mathrm{CO}+$ \\
Face_to_face & $\mathrm{A}+$ \\
Such_that & $-\mathrm{Pa} \& \mathrm{Ce}+$ \\
\hline
\end{tabular}

TABLE III

SOME ENTRIES IN THE POSTPROCESSING DICTIONARY

\section{EXPERIMENTS AND RESULTS}

One problem that we faced was that no gold standard is available for proper evaluation. And our purpose is not to compare with other grammars but to improve its coverage without disturbing the core grammar. This section describes the results of an experiment in which we applied our preprocessor to a randomly chosen subset of the WSJ corpus section 23. For these experiments, we used Kakkonen's evaluation tool ([12], [13]). Our purpose in doing this experiment was two fold.

\footnotetext{
${ }^{11}$ A dictionary consists of words and grammar that will have rules about how one word can be connected to the other words

${ }^{12}$ Note that it is a word based grammar
} 


\begin{tabular}{|l|r|}
\hline Sentences & 2416 \\
\hline Total parses & 1383369261 \\
\hline Parses/sentences & 572586 \\
\hline Sentences with a complete parse & 1404 \\
\hline An incomplete parse & 909 \\
\hline No parse & 103 \\
\hline Crashes & 1 \\
\hline An unique parse & 0 \\
\hline A complete unique parse & 0 \\
\hline Panics & 55 \\
\hline Coverage & 0.5808854 \\
\hline
\end{tabular}

TABLE IV

LINK PARSER COVERAGE EVALUATION RESULTS WITHOUT PRE- AND POSTPROCESSOR

\begin{tabular}{|l|r|}
\hline Sentences & 2416 \\
\hline Total parses & 1344183665 \\
\hline Parses/sentences & 556367 \\
\hline Sentences with a complete parse & 1756 \\
\hline An incomplete parse & 561 \\
\hline No parse & 99 \\
\hline Crashes & 1 \\
\hline An unique parse & 0 \\
\hline A complete unique parse & 0 \\
\hline Panics & 31 \\
\hline Coverage & 0.7265205 \\
\hline
\end{tabular}

TABLE V

LINK PARSER COVERAGE EVALUATION RESULTS WITH PRE- AND POSTPROCESSOR

We wanted to determine to what extent our preprocessor and postprocessor actually increase the converge of the parser by handling the problematic constructions. We also wanted to see whether our approach allows the end user to contribute to the development of the parsing system to make it more robust. Table IV gives the results without the preprocessor and the postprocessor, while Table $\mathrm{V}$ gives the results with them. As the table shows, there was a significant increase (14\%) in the coverage of the parser by using our simple approach. And we were able to achieve this relatively easily without causing any adverse effects.

There are still many issues that can cause problems. For example, we can take care of the topicalization constructions, but the problem is that it is hard to automatically identify the cases of topicalization. Similar is the case with extraposition.

\section{CONCLUSION}

In this paper we have proposed an approach to improve the Link Parser's coverage. The proposed approach has not only shown significant improvement but also allows further development by users not very familiar with the internals of the grammar. We first discussed various syntactic constructions that have not been handled by the parser and their frequency distribution in a representative corpus. In order to handle these constructions we introduced pre and postprocessors. The former identifies the cases where the parser fails to parse, while the latter provide the grammar to add link labels in such cases. We also showed why the obvious approach (increasing the grammar using additional rules or changing the grammar) does not easily work. Our approach allows a common user to contribute to the development of the parser with little knowledge of Link Grammar. Also, even though good statistical parsers may be available, there are applications like grammar checking where rule based parsers like the Link Parser may have an edge.

\section{ACKNOWLEDGEMENT}

Authors would like to acknowledge the guidance of Dr. Vineet Chaitanya whose suggestion originated this work. We convey our gratitude to him for his guidance, support and direction, especially for reading the drafts and giving feedback. Authors also sincerely thank Prof. Aditi Mukherjee of Osmania University for proof reading the paper.

\section{REFERENCES}

[1] D. Sleator and D. Temperley, "Parsing English with a link grammar," Arxiv preprint cmp-lg/9508004, 1995.

[2] D. Grinberg, J. Lafferty, and D. Sleator, "A robust parsing algorithm for link grammars," Arxiv preprint cmp-lg/9508003, 1995.

[3] G. Schneider, "A linguistic comparison of constituency, dependency and link grammar," Zurich, Switzerland: Licentiate Thesis, University of Zurich, 1998.

[4] Collins, M. J., "Head-driven statistical models for natural language parsing," in PhD thesis, University of Pennsylvania, 1999.

[5] M. Collins and N. Duffy, "Discriminative reranking for natural language parsing," Computational Linguistics, 2005.

[6] J. Hall, J. Nivre, and J. Nilsson, "Discriminative classifiers for deterministic dependency parsing," in Proceedings of the COLING/ACL 2006 Main Conference Poster Sessions, 2006, pp. 316-323.

[7] H. Gaifman, "Dependency systems and phrase-structure systems," Information and Control, pp. 304-337, 1965.

[8] J. Ouhalla, Introducing transformational grammar: from Principles and Parameters to Minimalism. Hodder Arnold, 1999.

[9] R. Hudson, Word grammar. Blackwell, 1984.

[10] B. Webber, A. Knott, and A. Joshi, "Multiple discourse connectives in a lexicalized grammar for discourse," STUDIES IN LINGUISTICS AND PHILOSOPHY, pp. 229-246, 2001.

[11] E. Miltsakaki, R. Prasad, A. Joshi, and B. Webber, "Annotating discourse connectives and their arguments," in Proceedings of the HLT/NAACL Workshop on Frontiers in Corpus Annotation, 2004, pp. 9-16.

[12] T. Kakkonen, "Framework and resources for natural language parser evaluation," Ph.D. dissertation, Department of Computer Science and Statistics, University of Joensuu.

[13] $\ldots$, "Robustness evaluation of two ccg, a pcfg and a link grammar parsers," Arxiv preprint arXiv:0801.3817, 2008. 